\title{
One-factorisations of complete graphs constructed in Desarguesian planes of certain odd square orders
}

\author{
Nicola Pace \\ Chair of Operations Research \\ Technical University of Munich \\ Munich, Germany \\ nicolaonline@libero.it
}

\author{
Angelo Sonnino \\ Dipartimento di Matematica, Informatica ed Economia \\ Università degli Studi della Basilicata \\ Potenza, Italy \\ angelo.sonnino@unibas.it
}

Submitted: Dec 10, 2018; Accepted: Jan 21, 2020; Published: Feb 7, 2020

(C) The authors. Released under the CC BY-ND license (International 4.0).

\begin{abstract}
A geometric construction of one-factorisations of complete graphs $K_{q(q-1)}$ is provided for the case when either $q=2^{d}+1$ is a Fermat prime, or $q=9$. This construction uses the affine group $\operatorname{AGL}(1, q)$, points and ovals in the Desarguesian plane $\mathrm{PG}\left(2, q^{2}\right)$ to produce one-factorisations of the complete graph $K_{q(q-1)}$.
\end{abstract}

Mathematics Subject Classifications: 05C70, 51E21, 05B25

\section{Introduction}

Let $K_{n}$ be the complete graph with an even number $n$ of vertices. A one-factor is a set of edges of $K_{n}$ such that every vertex of $K_{n}$ meets exactly one of its edges. A onefactorisation of $K_{n}$ is a partition of its edge set into $n-1$ disjoint one-factors.

One-factorisations of complete graphs have been studied in connection with Steiner triple systems and - more generally - Design theory [22]. They also have practical applications such as scheduling single round robin tournaments.

The systematic study of one-factorisations begun with the pioneering work of Rosa, Mendelsohn, Wallis and others; see for instance [4, 5, 6, 7, 13, 16, 17, 20, 22]. In some recent papers $[10,14,18]$, Gy. Kiss, G. Korchmáros and the authors used geometry in order to set up a procedure for the construction of (possibly new) families of one-factorisations based on nice geometric objects. The idea of using geometry for the construction of factorisations dates back to 2001 when it was first exploited on multigraphs; see for instance $[1,9,11,15,19]$. In this paper, we construct one-factorisations from ovals in a Desarguesian projective plane of odd square order, the order of which is either $9^{2}$, or the square of a prime of the form $2^{d}+1$.

Our notation and terminology are standard. In particular, the term partial onefactorisation stands for a set of one-factors of $K_{n}$ such that no two of them share an 
edge. Obviously, any subset of one-factors in a one-factorisation of $K_{n}$ is a partial onefactorisation. Background on one-factorisations of complete graphs is found in [21], and [17]. For a detailed account on ovals in finite projective planes; see [8].

The main result of this paper is a (mostly geometrical) construction of one-factorisations of the complete graph $K_{q(q-1)}$, where $q=2^{d}+1$ is a prime power.

Theorem 1. Let $q=2^{d}+1$ be a prime number. Let $\Sigma \cong \operatorname{AGL}(1, q)$ be the affine group represented by its sharply transitive action on the points of an oval $\mathcal{C}$ in $\mathrm{PG}\left(2, q^{2}\right) \backslash$ $\mathrm{PG}(2, q)$. There exists a one-factorisation

$$
\mathcal{F}=\left\{F_{1}, F_{1}^{\prime}, \ldots F_{\frac{q(q-3)}{2}}, F_{\frac{q(q-3)}{2}}^{\prime}, S_{1}, \ldots, S_{q-1}, R_{1}, \ldots, R_{q}\right\}
$$

of the complete graph $K_{q(q-1)}$ such that

- the union $F_{i} \cup F_{i}^{\prime}$ corresponds to a $\Sigma$-orbit of length $q(q-1)$, for $i=1, \ldots, \frac{q(q-3)}{2}$;

- $S_{i}$ corresponds to a $\Sigma$-orbit of length $\frac{q(q-1)}{2}$, for $i=1, \ldots, q-1$;

- $R_{i}$ corresponds to a set consisting of points in $\Sigma$-orbits of length $q(q-1)$ and points in $\mathrm{PG}(2, q)$ that are external to $\mathcal{C}$, for $i=1, \ldots, q$.

Theorem 2. Let $q=9$ and $\Sigma \cong \operatorname{AGL}(1, q)$ the affine group represented by its sharply transitive action on the points of an oval $\mathcal{C}$ in $\mathrm{PG}(2,81) \backslash \mathrm{PG}(2,9)$. Then there exists a one-factorisation

$$
\mathcal{F}=\left\{F_{1}, F_{1}^{\prime}, \ldots F_{27}, F_{27}^{\prime}, L_{1}, \ldots, L_{8}, R_{1}, \ldots, R_{9}\right\}
$$

of the complete graph $K_{72}$ such that

- the union $F_{i} \cup F_{i}^{\prime}$ corresponds to a $\Sigma$-orbit of length 72 , for $i=1, \ldots, 27$;

- $L_{i}$ corresponds to a vertical line in $\mathrm{PG}(2,81)$, for $i=1, \ldots, 8$;

- $R_{i}$ corresponds to a set with 32 points in $\Sigma$-orbits of length 72 and 4 points on the vertical line $X=0$ in $\mathrm{PG}(2,81)$, for $i=1, \ldots, 9$.

Note that if $q=2^{d}+1$ is a prime power, then either $q=9$, or $q$ is a so-called Fermat prime, where $d$ is a power of 2 . Although number theoretic implications are out of the scope of this paper, it is worth mentioning that only finitely many Fermat primes are known, and whether Fermat primes form a finite or an infinite family is still an open question.

The case $q$ prime (Theorem 1 ) is dealt with in Section 3, and the special case $q=9$ (Theorem 2) is considered in subsection 4.2. 


\section{Preliminaries}

For an odd prime power $q=p^{h}$, let $\Omega$ be an oval in the projective plane $\pi=\operatorname{PG}\left(2, q^{2}\right)$ and $\pi_{0}=\operatorname{PG}(2, q)$ a Baer subplane of $\pi$ such that $\pi_{0} \cap \Omega \neq \emptyset$. From [12, Lemma 4] we know that

- $\left|\pi_{0} \cap \Omega\right|=q+1$ and $\Omega_{0}=\pi_{0} \cap \Omega$ is an oval of $\pi_{0}$.

- every line of $\pi_{0}$ meets $\Omega$ at some point, and those missing $\Omega_{0}$ are secants to $\Omega$,

- no point of $\pi_{0}$ is internal to $\Omega$.

There are exactly $\frac{q^{2}\left(q^{2}+1\right)}{2}$ points of $\mathrm{PG}\left(2, q^{2}\right)$ which are external to $\Omega$, each of them lying on two tangents to $\Omega$. We denote the set of all external points to $\Omega$ with $E(\Omega)$.

Let $S$ be a set of points in $\operatorname{PG}\left(2, q^{2}\right)$ and $T$ be a set of tangents to $\Omega$. Then $S$ is said to have the tangent property with respect to $T$ if and only if for every $\ell \in T$ the condition $|\ell \cap S| \leqslant 1$ holds. Remark that this definition generalises the notion of tangent property introduced in [14].

The action of the group $\operatorname{PGL}(2, q)$ on $\Omega$ yields two orbits: one of them is $\Omega_{0}$ itself, while the other is $\Omega^{*}=\Omega \backslash \Omega_{0}$. We call real tangents the tangents to $\Omega$ at points in $\Omega_{0}$, and complex tangents the others.

Our aim is to construct a geometric one-factorisation of the complete graph $K_{n}$, with $n=q^{2}-q$, whose vertices are represented by the points, and edges by the chords of $\Omega^{*}$. Following to [14], we are looking for a partition of the points of $E^{\prime}=E(\Omega) \backslash \pi$ into subsets consisting of $\frac{q^{2}-q}{2}$ points, each of them with the tangent property, henceforth with respect to the set of complex tangents to $\Omega$.

Consider an affine frame where the oval $\Omega$ is a parabola $\mathcal{C}$ of affine equation

$$
Y=X^{2}
$$

in $\mathrm{AG}\left(2, q^{2}\right)$. One can label the affine points $P\left(\xi, \xi^{2}\right)$ in $\mathrm{AG}\left(2, q^{2}\right)$ with the corresponding element $\xi \in \mathrm{GF}\left(q^{2}\right)$ and denote it with $P_{\xi}$. Then, the equation of the tangent to $\Omega$ at one of its points $P_{\xi}$ is

$$
Y=2 \xi X-\xi^{2}
$$

Hence, the points of $E^{\prime}$ are in one-to-one correspondence with pairs $\left\{\xi_{1}, \xi_{2}\right\}$, where $\xi_{1}, \xi_{2} \in$ $\mathrm{GF}\left(\mathrm{q}^{2}\right) \backslash \mathrm{GF}(\mathrm{q})$. Namely, the pair $\left\{\xi_{1}, \xi_{2}\right\}$ corresponds to the external point $\left(\frac{\xi_{1}+\xi_{2}}{2}, \xi_{1} \xi_{2}\right)$ and vice versa.

One can look at the action of the affine group $\Sigma \cong \operatorname{AGL}(1, q)$ on the points of $\mathcal{C}$ and $E^{\prime}$ realised in the following way. For $a \in \mathrm{GF}(\mathrm{q})$, define the map $\varphi_{a}$ as

$$
\varphi_{a}(x, y)=\left(x+a, y+2 a x+a^{2}\right)
$$

and the group $\Phi=\left\langle\varphi_{a} \mid a \in \operatorname{GF}(q)\right\rangle$. Similarly, for $\lambda \in \operatorname{GF}(q) \backslash\{0\}$, define

$$
\psi_{\lambda}(x, y)=\left(\lambda x, \lambda^{2} y\right)
$$


and the group $\Psi=\left\langle\psi_{\lambda} \mid \lambda \in \mathrm{GF}(q) \backslash\{0\}\right\rangle$. Then let

$$
\Sigma=\left\langle\varphi_{a} \psi_{\lambda} \mid a \in \mathrm{GF}(q), \lambda \in \mathrm{GF}(q) \backslash\{0\}\right\rangle .
$$

It is not hard to see that $\Sigma=\Psi \ltimes \Phi \cong \operatorname{AGL}(1, q)$, and its action on the points of $\mathcal{C}$ in $\operatorname{AG}\left(2, q^{2}\right) \backslash \operatorname{AG}(2, q)$ is sharply transitive. Furthermore, the group $\Sigma$ partitions the set $E^{\prime}$ in $\Sigma$-orbits of length $n=q(q-1)$ or $n / 2=q(q-1) / 2$, which are called long and short orbits, respectively; see Lemma 5 for a formal proof. Note that the notion of long and short orbit is consistent with the notion of short and long edges used in the usual constructions with starters; see for instance [2].

A geometric characterisation of the points of $E^{\prime}$ and the $\Sigma$-orbits is provided by the following sequence of lemmas.

Lemma 3. Every external point in $E^{\prime}$ belongs to a unique parabola $\mathcal{C}_{t}$ of affine equation $Y=X^{2}-t$, where $t=s^{2}$ is a nonzero square in $\operatorname{GF}\left(q^{2}\right)$.

Proof. Let $P_{\xi_{1}}\left(\xi_{1}, \xi_{1}^{2}\right)$ be a point of $\mathcal{C}$, where $\xi_{1} \in \mathrm{GF}\left(q^{2}\right) \backslash \mathrm{GF}(q)$. The tangent to $\mathcal{C}$ at $P_{\xi_{1}}$ has affine equation

$$
t_{\xi_{1}}: Y=2 \xi_{1} X-\xi_{1}^{2} .
$$

The intersection $t_{\xi_{1}} \cap \mathcal{C}_{t}$ is constituted by points of type $\left(x, x^{2}-t\right)$, where $x$ is such that $\left(x-\xi_{1}\right)^{2}=t$. Thus, $t=s^{2}$ must be a nonzero square and the points of intersection are the points $\left(\xi_{1}-s, \xi_{1}^{2}-2 s \xi_{1}\right),\left(\xi_{1}+s, \xi_{1}^{2}+2 s \xi_{1}\right)$.

Lemma 4. Let $\mathcal{C}_{t}$ be an external parabola. Then the following hold:

1. $\varphi_{a}\left(\mathcal{C}_{t}\right)=\mathcal{C}_{t}$, for all $a \in \operatorname{GF}(q)$;

2. $\psi_{\lambda}\left(\mathcal{C}_{t}\right)=\mathcal{C}_{\lambda^{2} t}$, for all $\lambda \in \mathrm{GF}(q) \backslash\{0\}$.

Lemma 5. The group $\Sigma$ partitions the set $E^{\prime}$ in $\Sigma$-orbits of length either $q(q-1)$, or $\frac{q(q-1)}{2}$. Furthermore, the following hold:

1. short $\Sigma$-orbits have the tangent property, i.e. they correspond to one-factors;

2. long $\Sigma$-orbits have the 2-tangent property, that is, every tangent to $\mathcal{C}$ at a point $P_{\xi}$, for $\xi \in \mathrm{GF}\left(q^{2}\right) \backslash \mathrm{GF}(q)$, intersects a $\Sigma$-orbits in exactly two points.

Proof. Let $P\left(\frac{\xi_{1}+\xi_{2}}{2}, \xi_{1} \xi_{2}\right) \in E^{\prime}$ be the intersection of the tangents $t_{\xi_{1}}$ and $t_{\xi_{2}}$, where $\xi_{1}, \xi_{2} \in \mathrm{GF}\left(q^{2}\right) \backslash \mathrm{GF}(q)$ and $\xi_{1} \neq \xi_{2}$.

Let $\sigma=\psi_{\lambda} \circ \varphi_{a} \in \Sigma$ be such that $\sigma(P)=P$. Then $\lambda\left(\xi_{1}+a\right)=\xi_{2}, \lambda\left(\xi_{2}+a\right)=\xi_{1}$ and this implies that either $\sigma=1_{\Sigma}$, or $\sigma=\psi_{-1} \circ \varphi_{a}$ and $\xi_{1}+\xi_{2}=-a$. The latter case occurs only when $P^{\Sigma}$ is a short orbit and every short orbit contains some point of the form $(0,-t)$, where $t \neq 0$ is a square in $\operatorname{GF}\left(q^{2}\right)$.

Since the group $\operatorname{AGL}(1, q)$ is transitive on the elements of $\operatorname{GF}\left(q^{2}\right) \backslash \operatorname{GF}(q)$, every complex tangent intersects a $\Sigma$-orbit $P^{\Sigma}$ in at least one point. Furthermore, there are $q(q-1)$ complex tangents and every point of $P^{\Sigma}$ is on at most two tangents.

Then, since the length of a short orbit is $\frac{q(q-1)}{2}$, every complex tangent intersects it in exactly one point. Similarly, the length of a long orbit is $q(q-1)$ and every complex tangent must intersect it in exactly two points. 
Finally, the following lemma is needed for the proof in the next section.

Lemma 6. Let $n$ be a positive integer. There exist $2 n$ distinct integers

$$
0 \leqslant x_{1}, \ldots, x_{n}, y_{1}, \ldots, y_{n} \leqslant 2 n
$$

such that $x_{i}-y_{i}=i$, for all $i=1, \ldots, n$.

Proof. For $k=1, \ldots,\left\lceil\frac{n}{2}\right\rceil$, take the pairs

$$
p_{k}^{(l)}=[n-k+1, k-1]
$$

and for $k=\left\lceil\frac{n}{2}\right\rceil+1, \ldots, n$, take the pairs

$$
p_{k}^{(r)}=[n+k, 2 n-k+1] .
$$

Note that, if $n$ is even (resp. odd) then the pairs $p_{k}^{(l)}$ produce all even (resp. odd) differences:

$$
n-2 k+2, \text { for } k=1, \ldots,\left\lceil\frac{n}{2}\right\rceil .
$$

Similarly, if $n$ is even (resp. odd) the pairs $p_{k}^{(r)}$ produce all odd (resp. even) differences:

$$
2 k-n-1, \text { for } k=\left\lceil\frac{n}{2}\right\rceil+1, \ldots, n .
$$

The result follows.

\section{Proof of Theorem 1}

In this section, we provide a construction for $q=2^{d}+1, q$ prime. We start from the partition of $E^{\prime}$ in $\Sigma$-orbits. By Lemma 5, the short orbits have the tangent property and there is nothing to prove. Furthermore, each long orbit has the 2-tangent property and can be partitioned into polygons - that is, cycles in the underlying graph - where two vertices are adjacent if and only if the corresponding points are on the same tangent line. If the number of vertices is even, then we can colour the vertices with two colours-red and green - and each of these good orbits produces two one-factors.

Let $P\left(\frac{\xi_{1}+\xi_{2}}{2}, \xi_{1} \xi_{2}\right)$ be a point in a long orbit and $\sigma=\psi_{\lambda} \circ \varphi_{a}$ be such that $\xi_{2}=\lambda\left(\xi_{1}+a\right)$. Then the polygon that contains $P$ has exactly $k=|\sigma|$ vertices and it can be represented by the following ordered sequence of pairs:

$$
\left[\left\{\xi_{1}, \sigma\left(\xi_{1}\right)\right\},\left\{\sigma\left(\xi_{1}\right), \sigma^{2}\left(\xi_{1}\right)\right\}, \ldots,\left\{\sigma^{k-1}\left(\xi_{1}\right), \xi_{1}\right\}\right] .
$$

The condition $q=2^{d}+1$ implies that $P^{\Sigma}$ is a "good orbit" if and only if $\lambda \neq 1$. Then $k$ is even and the polygon can be easily coloured as follows:

$$
\begin{aligned}
\mathcal{G} & =\left\{\left\{\xi_{1}, \sigma\left(\xi_{1}\right)\right\},\left\{\sigma^{2}\left(\xi_{1}\right), \sigma^{3}\left(\xi_{1}\right)\right\}, \ldots,\left\{\sigma^{k-2}\left(\xi_{1}\right), \sigma^{k-1}\left(\xi_{1}\right)\right\}\right\}, \\
\mathcal{R} & =\left\{\left\{\sigma\left(\xi_{1}\right), \sigma^{2}\left(\xi_{1}\right)\right\},\left\{\sigma^{2}\left(\xi_{1}\right), \sigma^{3}\left(\xi_{1}\right)\right\}, \ldots,\left\{\sigma^{k-1}\left(\xi_{1}\right), \xi_{1}\right\}\right\} .
\end{aligned}
$$


Note that, if $P^{\Sigma}$ is a long orbit and the order of $\sigma$ is even, then $P$ is necessarily on a conic $\mathcal{C}_{t}$, where $t=s^{2}$ and $s \in \mathrm{GF}\left(q^{2}\right) \backslash \mathrm{GF}(q)$. In this case, it can be easily checked that every orbit $P^{\Phi}$ has the tangent property.

The orbits that split into polygons with an odd number of vertices cannot be coloured with two colours and are called bad orbits. These orbits exist because of the subgroups of $\Sigma$ of odd order. If $q$ is a prime number such that $q=2^{d}+1$, then each of the bad orbits is the union of exactly $(q-1) q$-gons. Each of these $q$-gons corresponds to a set of pairs of the type

$$
\{\{x, x+b\},\{x+b, x+2 b\}, \ldots,\{x-b, x\}\},
$$

where $x \in \mathrm{GF}\left(q^{2}\right) \backslash \mathrm{GF}(q)$ and $b \in \mathrm{GF}(q) \backslash\{0\}$. From a simple counting argument, we can also note that there are exactly $\frac{q-1}{2}$ bad orbits.

Every point $P$ in a bad orbit must lie on a conic $\mathcal{C}_{t}$, where $t=s^{2}$ and $s \in \operatorname{GF}(q) \backslash\{0\}$, and the union of all bad orbits is equal to the disjoint union of all sets $E^{\prime} \cap \mathcal{C}_{t}$. Furthermore, unlike the previous case, the orbit $P^{\Phi}$ does not have the tangent property.

Let $w$ be a primitive element of $\operatorname{GF}\left(q^{2}\right)$. Then, each of the sets $E^{\prime} \cap \mathcal{C}_{t}$ can be further partitioned in $\frac{(q-1)}{2}$ pairs of $q$-gons as follows:

$$
\begin{aligned}
E^{\prime} \cap C_{t} & =\left\{\left(x, x^{2}-t\right) \mid x \in \mathrm{GF}\left(q^{2}\right) \backslash \mathrm{GF}(q)\right\} \\
& =\bigcup_{\lambda=1}^{(q-1) / 2} P_{\lambda w, t} \cup P_{-\lambda w, t}=\bigcup_{\lambda=1}^{(q-1) / 2} P_{\lambda w, t} \cup P_{\lambda w^{q}, t},
\end{aligned}
$$

where every orbit $P_{x, t}=\left(x, x^{2}-t\right)^{\Phi}$ is one the $q$-gons embedded in $\mathcal{C}_{t}$. Note that every set $E^{\prime} \cap \mathcal{C}_{t}$ is a $q(q-1)$-arc and has the 2-tangent property.

Take the short orbit $O_{0}$ corresponding to the pairs $\left\{\left\{x, x^{q}\right\} \mid x \in \operatorname{GF}\left(q^{2}\right) \backslash \operatorname{GF}(q)\right\}$. In the remainder of this section we show how to replace a point from each $q$-gon contained in $E^{\prime} \cap \mathcal{C}_{t}$ with a point from $O_{0}$ so that two $\frac{q(q-1)}{2}$-sets with the tangent property are obtained.

Let $t=s^{2}$ for $s \in \mathrm{GF}(q) \backslash\{0\}$, and $P:=(x+s, x(x+2 s)), Q:=\left(x^{q}+s, x^{q}\left(x^{q}+2 s\right)\right)$ be the points corresponding to the pairs $\{x, x+2 s\},\left\{x^{q}, x^{q}+2 s\right\}$. The points $P, Q$ belong to two paired $q$-gons $P_{\lambda w, t}, P_{\lambda w^{q}, t}$, where

$$
\begin{aligned}
P_{\lambda w, t} & =\left[P, P_{1}=\varphi_{2 s}(P), \ldots, P_{q-1}=\varphi_{(2 s)^{q-1}}(P)\right], \\
P_{\lambda w^{q}, t} & =\left[Q, Q_{1}=\varphi_{2 s}(Q), \ldots, Q_{q-1}=\varphi_{(2 s)^{q-1}}(Q)\right] .
\end{aligned}
$$

We replace $P, Q$, that is, the pairs $\{x, x+2 s\},\left\{x^{q}, x^{q}+2 s\right\}$, with two points $R_{1}, R_{2}=$ $\varphi_{2 s}\left(R_{1}\right)$ from $O_{0}$, where $R_{1}$ and $R_{2}$ correspond to the pairs $\left\{x, x^{q}\right\},\left\{x+2 s, x^{q}+2 s\right\}$, respectively. In affine coordinates, we have

$$
R_{1}:=\left(\frac{x+x^{q}}{2}, x^{q+1}\right), \quad R_{2}:=\left(\frac{x+x^{q}}{2}+2 s,(x+2 s)^{q+1}\right) .
$$

Then, we can colour $R_{1}, R_{2}$ and the remaining points of the $q$-gons with two colors $\mathcal{G}$ (green) and $\mathcal{R}$ (red) as follows:

$$
\begin{aligned}
\mathcal{G} & =\left\{R_{1}, P_{1}, P_{3}, \ldots, P_{q-2}, Q_{1}, Q_{3}, \ldots, Q_{q-2}\right\} \\
\mathcal{R} & =\varphi_{2 s}(\mathcal{G})=\left\{R_{2}, P_{2}, P_{4}, \ldots, P_{q-1}, Q_{2}, Q_{4}, \ldots, Q_{q-1}\right\}
\end{aligned}
$$


see Figure 1. Note that in our argument we heavily used the fact that $(x+2 s)^{q}=x^{q}+2 s$.

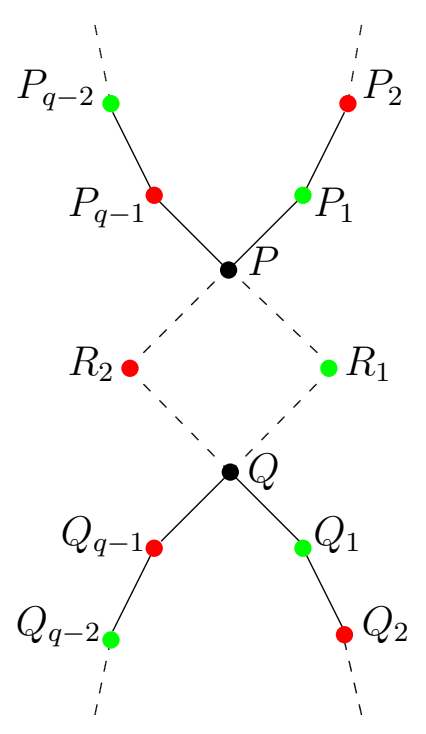

Figure 1: A pair of $q$-gons embedded in $C_{t}$

We want to use this strategy for all $\frac{q-1}{2}$ conics $C_{t}$, for $t=s^{2}$ and $s \in \mathrm{GF}(q) \backslash\{0\}$. We have a total of $\frac{(q-1)^{2}}{2} q$-gons in bad orbits. For each pair of $q$-gons $P_{\lambda w, t}, P_{\lambda w^{q}, t}$ we need to be able to find two suitable points $R_{1}, R_{2}$, corresponding to two pairs of the type $\left\{x, x^{q}\right\},\left\{x+2 s, x^{q}+2 s\right\}$. There seems to be enough points, more precisely, there are $\frac{q(q-1)}{2}$ of them in $O_{0}$. However, we have to make sure that once we use two points $R_{1}, R_{2}$, they are not used with any other $q$-gon.

The orbit $O_{0}$ can be partitioned in the disjoint union of sets $\pi_{0} \cap C_{r}$, for $r=z^{2} \in \operatorname{GF}(q)$ and $z \in \operatorname{GF}\left(q^{2}\right) \backslash \operatorname{GF}(q)$. Note that $z^{q}=-z$. By Lemma 6, for every $t=s^{2}$, with $s \in \mathrm{GF}(q)$, we can find $u_{t}, v_{t}$ such that $v_{t}-u_{t}=2 s$ and $\left|\bigcup_{t}\left\{u_{t}, v_{t}\right\}\right|=q-1$.

Let $\pi_{0} \cap C_{r}$ be one of these sets from $O_{0}$. The points from $\mathcal{C}_{r}$

$$
R_{1}\left(u_{t}, u_{t}^{2}-r\right), \quad R_{2}\left(v_{t}, v_{t}^{2}-r\right)
$$

corresponding to the pairs $\left\{u_{t}+z, u_{t}-z\right\}=\left\{u_{t}+z, u_{t}+z^{q}\right\},\left\{v_{t}+z, v_{t}-z\right\}=\left\{v_{t}+z, v_{t}+z^{q}\right\}$, can be used to replace the points from $\mathcal{C}_{t}$

$$
P\left(v_{t}+s+z,\left(v_{t}+s+z\right)^{2}-t\right), \quad Q\left(v_{t}+s+z^{q},\left(v_{t}+s+z^{q}\right)^{2}-t\right),
$$

corresponding to the pairs $\left\{u_{t}+z, v_{t}+z\right\},\left\{u_{t}+z^{q}, v_{t}+z^{q}\right\}$. Remark that the points $P, Q$ lie on two paired q-gons, say $P_{\lambda w, t} \cup P_{\lambda w^{q}, t}=P_{z, t} \cup P_{z^{q}, t}$. Furthermore, $C_{\bar{r}}$ can be written as $C_{\bar{\lambda}^{2} r}$, for $\bar{\lambda} \neq \pm 1$, and the points

$$
\bar{R}_{1}\left(u_{t}, u_{t}^{2}-\bar{r}\right), \quad \bar{R}_{2}\left(v_{t}, v_{t}^{2}-\bar{r}\right) \in C_{\bar{r}}
$$

replace two points in $C_{t}$ that must be in a different pair of $q$-gons. This completes the proof. 


\section{$4 \quad$ Examples}

In 4.1 we illustrate the construction in Section 3 for the case $q=5$. We obtain a partition into 10-sets with the tangent property that corresponds to a (rigid) 1-factorisation of the complete graph with 20 vertices $K_{20}$.

In 4.2 we consider the special case $q=9$, which is obtained by a slight modification of the main construction.

\subsection{Case $q=5$}

Let $w$ be a primitive element of $\mathrm{GF}(25)$ such that $w^{2}=w+3$.

Consider the conic $\mathcal{C}$ of equation $Y=X^{2}$ in $\mathrm{PG}(2,25)$. There are twelve $\Sigma$-orbits: 5 short orbits, 5 good (long) orbits, and 2 bad (long) orbits. The points in bad orbits are contained in the conics

$$
\mathcal{C}_{1}: Y=X^{2}-1, \quad \mathcal{C}_{4}: Y=X^{2}-4
$$

The conic $\mathcal{C}_{1}$ contains the following four pentagons:

$$
\begin{aligned}
P_{w, 1} & =\left[\left(w, w^{15}\right),\left(w^{15}, 1\right),\left(w^{17}, w^{3}\right),\left(w^{22}, w^{16}\right),\left(w^{2}, w^{8}\right)\right], \\
P_{w^{5}, 1} & =\left[\left(w^{5}, w^{3}\right),\left(w^{3}, 1\right),\left(w^{13}, w^{15}\right),\left(w^{14}, w^{8}\right),\left(w^{10}, w^{16}\right)\right], \\
P_{2 w, 1} & =\left[\left(w^{7}, w^{5}\right),\left(w^{4}, w^{7}\right),\left(w^{21}, 2\right),\left(w^{8}, w^{11}\right),\left(w^{23}, w\right)\right], \\
P_{2 w^{5}, 1} & =\left[\left(w^{11}, w\right),\left(w^{20}, w^{11}\right),\left(w^{9}, 2\right),\left(w^{16}, w^{7}\right),\left(w^{19}, w^{5}\right)\right] .
\end{aligned}
$$

The conic $\mathcal{C}_{4}$ contains the following four pentagons:

$$
\begin{aligned}
P_{w, 2} & =\left[\left(w, w^{17}\right),\left(w^{17}, w^{13}\right),\left(w^{2}, w^{23}\right),\left(w^{15}, 3\right),\left(w^{22}, w^{19}\right)\right], \\
P_{w^{5}, 2} & =\left[\left(w^{5}, w^{13}\right),\left(w^{13}, w^{17}\right),\left(w^{10}, w^{19}\right),\left(w^{3}, 3\right),\left(w^{14}, w^{23}\right)\right], \\
P_{2 w, 2} & =\left[\left(w^{7}, w^{3}\right),\left(w^{21}, 4\right),\left(w^{23}, w^{15}\right),\left(w^{4}, w^{4}\right),\left(w^{8}, w^{20}\right)\right], \\
P_{2 w^{5}, 2} & =\left[\left(w^{11}, w^{15}\right),\left(w^{9}, 4\right),\left(w^{19}, w^{3}\right),\left(w^{20}, w^{20}\right),\left(w^{16}, w^{4}\right)\right] .
\end{aligned}
$$

The short orbit $O_{0}$ can be written as the disjoint union

$$
O_{0}=\left(\pi_{0} \cap C_{2}\right) \cup\left(\pi_{0} \cap C_{3}\right),
$$

where

$$
\pi_{0} \cap C_{2}=\{(0,3),(1,4),(2,2),(3,2),(4,4)\}
$$

and

$$
\pi_{0} \cap C_{3}=\{(0,2),(1,3),(2,1),(3,1),(4,3)\} .
$$

Take $u_{1}=4, v_{1}=3, u_{2}=2, v_{2}=0$. Note that $u_{1}-v_{1}=1$ and $u_{2}-v_{2}=2$. Then we can consider the following pairs of points from $O_{0}$ :

(i) $(3,2),(4,4)$; 
(ii) $(3,1),(4,3)$;

(iii) $(0,3),(2,2)$;

(iv) $(0,2),(2,1)$.

The points from the pairs (i) and (ii) will be used to replace points from the conic $\mathcal{C}_{4}$, while the points from the pairs (iii) and (iv) will be used to replace points from $\mathcal{C}_{1}$.

The points $R_{1}:=(4,4), R_{2}:=(3,2)=\varphi_{4}\left(R_{1}\right)$ correspond to the pairs $\left\{w^{14}, w^{22}\right\}$, Note that $w^{22}+4=w$ and $w^{14}+4=w^{5}$, hence $R_{1}$ and $R_{2}$ replace the points

$$
P:=\left(w^{2}, w^{23}\right), \quad Q:=\left(w^{10}, w^{19}\right),
$$

from the pentagons $P_{w, 2}, P_{w^{5}, 2}$ and corresponding to the pairs $\left\{w, w^{22}\right\}$, $\left\{w^{5}, w^{14}\right\}$, respectively. Similarly, the points $R_{1}^{\prime}:=(4,3), R_{2}^{\prime}:=(3,1)$ correspond to the pairs $\left\{w^{19}, w^{23}\right\},\left\{w^{4}, w^{20}\right\}$ and replace the points $P^{\prime}:=\left(w^{7}, w^{3}\right), Q^{\prime}:=\left(w^{11}, w^{15}\right)$ from the pentagons $P_{2 w, 2}, P_{2 w^{5}, 2}$.

Then, following the procedure from Section 3, we obtain two 10-sets $\mathcal{F}_{1}=\mathcal{G}, \mathcal{F}_{2}=\mathcal{R}$ with the tangent property:

$$
\begin{aligned}
\mathcal{G} & =\left\{R_{1}, \varphi_{2}(P), \varphi_{4}(P), \varphi_{2}(Q), \varphi_{4}(Q), R_{1}^{\prime}, \varphi_{2}\left(P^{\prime}\right), \varphi_{4}\left(P^{\prime}\right), \varphi_{2}\left(Q^{\prime}\right), \varphi_{4}\left(Q^{\prime}\right)\right\}, \\
\mathcal{R} & =\varphi_{4}(\mathcal{G}) .
\end{aligned}
$$

Following the same procedure, we can replace the points $\left(w^{2}, w^{8}\right) \in P_{w, 1}$ and $\left(w^{10}, w^{16}\right) \in$ $P_{w^{5}, 1}$ with $(0,3)$ and $(2,2)$; then the points $\left(w^{7}, w^{5}\right) \in P_{2 w, 1}$ and $\left(w^{11}, w\right) \in P_{2 w^{5}, 1}$ with $(0,2)$ and $(2,1)$. We obtain two further 10 -sets $\mathcal{F}_{3}, \mathcal{F}_{4}$ with the tangent property:

$$
\begin{aligned}
\mathcal{F}_{3}= & \left\{(0,3),\left(w, w^{15}\right),\left(w^{17}, w^{3}\right),\left(w^{5}, w^{3}\right),\left(w^{13}, w^{15}\right),\right. \\
& \left.(0,2),\left(w^{4}, w^{7}\right),\left(w^{8}, w^{11}\right),\left(w^{20}, w^{11}\right),\left(w^{16}, w^{7}\right)\right\}, \\
\mathcal{F}_{4}= & \varphi_{2}\left(\mathcal{F}_{3}\right) .
\end{aligned}
$$

Another four 10-sets with the tangent property consist of the following short orbits:

$$
\mathcal{F}_{5}=\left(0, w^{2}\right)^{\Sigma}, \mathcal{F}_{6}=\left(0, w^{4}\right)^{\Sigma}, \mathcal{F}_{7}=\left(0, w^{8}\right)^{\Sigma}, \mathcal{F}_{8}=\left(0, w^{10}\right)^{\Sigma} .
$$

The five (long) good orbits

$$
O_{1}=(w, 2)^{\Sigma}, O_{2}=(w, w)^{\Sigma}, O_{3}=\left(w, w^{8}\right)^{\Sigma}, O_{4}=\left(w, w^{14}\right)^{\Sigma}, O_{5}=\left(w, w^{19}\right)^{\Sigma}
$$

split into polygons with an even number of edges.

The orbit $O_{1}$ splits into five quadrangles:

$$
\begin{aligned}
& \mathcal{Q}_{1}=\left[(w, 2),\left(w^{16}, w^{11}\right),\left(w^{14}, w^{22}\right),\left(w^{21}, w^{17}\right)\right], \\
& \mathcal{Q}_{2}=\left[\left(w^{2}, w^{22}\right),\left(w^{4}, w^{11}\right),\left(w^{13}, 2\right),\left(w^{9}, w^{17}\right)\right], \\
& \mathcal{Q}_{3}=\left[\left(w^{3}, w^{5}\right),\left(w^{7}, 3\right),\left(w^{22}, w^{23}\right),\left(w^{20}, w^{10}\right)\right], \\
& \mathcal{Q}_{4}=\left[\left(w^{5}, w^{16}\right),\left(w^{11}, w^{4}\right),\left(w^{17}, w^{16}\right),\left(w^{23}, w^{4}\right)\right], \\
& \mathcal{Q}_{5}=\left[\left(w^{8}, w^{10}\right),\left(w^{10}, w^{23}\right),\left(w^{19}, 3\right),\left(w^{15}, w^{5}\right)\right] .
\end{aligned}
$$


Then, $O_{1}$ can be partitioned into two 10 -sets $\mathcal{F}_{9}$ and $\mathcal{F}_{10}$ with the tangent property:

$$
\begin{aligned}
\mathcal{F}_{9}=\{ & (w, 2),\left(w^{14}, w^{22}\right),\left(w^{2}, w^{22}\right),\left(w^{13}, 2\right),\left(w^{3}, w^{5}\right) \\
& \left.\left(w^{22}, w^{23}\right),\left(w^{5}, w^{16}\right),\left(w^{17}, w^{16}\right),\left(w^{8}, w^{10}\right),\left(w^{19}, 3\right)\right\} \\
\mathcal{F}_{10}= & \left(w^{16}, w^{11}\right),\left(w^{21}, w^{17}\right),\left(w^{4}, w^{11}\right),\left(w^{13}, 2\right),\left(w^{7}, 3\right) \\
& \left.\left(w^{20}, w^{10}\right),\left(w^{11}, w^{4}\right),\left(w^{23}, w^{4}\right),\left(w^{10}, w^{23}\right),\left(w^{15}, w^{5}\right)\right\} .
\end{aligned}
$$

In the same way, the orbits $O_{2}, O_{3}, O_{4}$ and $O_{5}$ split into quadrangles and produce the following 10-sets with the tangent property:

$$
\begin{aligned}
\mathcal{F}_{11}=\{ & (w, w),\left(w^{5}, w^{5}\right),\left(w^{2}, w^{21}\right),\left(w^{10}, w^{9}\right),\left(w^{3}, 4\right), \\
& \left.\left(w^{15}, 4\right),\left(w^{8}, w^{9}\right),\left(w^{16}, w^{21}\right),\left(w^{14}, w^{21}\right),\left(w^{22}, w^{9}\right)\right\}, \\
\mathcal{F}_{12}=\left\{\left(w^{4}, w^{21}\right),\left(w^{20}, w^{9}\right),\left(w^{7}, w^{13}\right),\left(w^{11}, w^{17}\right),\left(w^{9}, 1\right),\right. & \left.\left(w^{21}, 1\right),\left(w^{13}, w\right),\left(w^{17}, w^{5}\right),\left(w^{19}, w^{13}\right),\left(w^{23}, w^{17}\right)\right\}, \\
\mathcal{F}_{13}=\{ & \left(w, w^{8}\right),\left(w^{13}, w^{8}\right),\left(w^{2}, w^{19}\right),\left(w^{3}, w\right),\left(w^{4}, w^{2}\right), \\
& \left.\left(w^{11}, 3\right),\left(w^{5}, 2\right),\left(w^{22}, w^{14}\right),\left(w^{10}, w^{14}\right),\left(w^{17}, 2\right)\right\} \\
\mathcal{F}_{14}=\left\{\left(w^{7}, w^{20}\right),\left(w^{19}, w^{20}\right),\left(w^{16}, w^{2}\right),\left(w^{23}, 3\right),\left(w^{14}, w^{19}\right),\right. & \left.\left(w^{15}, w\right),\left(w^{8}, w^{7}\right),\left(w^{9}, w^{13}\right),\left(w^{20}, w^{7}\right),\left(w^{21}, w^{13}\right)\right\}, \\
\mathcal{F}_{15}=\{ & \left(w, w^{14}\right),\left(w^{3}, w^{9}\right),\left(w^{2}, 1\right),\left(w^{5}, w^{23}\right),\left(w^{7}, w^{2}\right), \\
& \left.\left(w^{9}, w^{21}\right),\left(w^{4}, w^{14}\right),\left(w^{16}, w^{14}\right),\left(w^{13}, w^{14}\right),\left(w^{15}, w^{9}\right)\right\}, \\
\mathcal{F}_{16}=\{ & \left(w^{8}, 4\right),\left(w^{11}, w^{11}\right),\left(w^{19}, w^{2}\right),\left(w^{21}, w^{21}\right),\left(w^{14}, 1\right), \\
& \left.\left(w^{17}, w^{23}\right),\left(w^{10}, w^{2}\right),\left(w^{22}, w^{2}\right),\left(w^{20}, 4\right),\left(w^{23}, w^{11}\right)\right\}, \\
\mathcal{F}_{17}=\{ & \left(w, w^{19}\right),\left(w^{10}, 1\right),\left(w^{2}, w^{10}\right),\left(w^{14}, w^{10}\right),\left(w^{3}, w^{21}\right), \\
& \left.\left(w^{17}, w^{22}\right),\left(w^{5}, w^{22}\right),\left(w^{15}, w^{21}\right),\left(w^{11}, w^{10}\right),\left(w^{21}, w^{9}\right)\right\}, \\
\mathcal{F}_{18}=\{ & \left(w^{9}, w^{9}\right),\left(w^{23}, w^{10}\right),\left(w^{8}, w^{22}\right),\left(w^{20}, w^{22}\right),\left(w^{4}, 4\right), \\
& \left.\left(w^{19}, w^{7}\right),\left(w^{7}, w^{7}\right),\left(w^{16}, 4\right),\left(w^{13}, w^{19}\right),\left(w^{22}, 1\right)\right\} .
\end{aligned}
$$

The last 10 -set $\mathcal{F}_{19}$ is uniquely determined by the previous ones:

$$
\begin{aligned}
\mathcal{F}_{19}=\{ & (1,3),(1,4),\left(w^{2}, w^{23}\right),\left(w^{2}, w^{8}\right),\left(w^{7}, w^{3}\right),\left(w^{7}, w^{5}\right), \\
& \left.\left(w^{10}, w^{16}\right),\left(w^{10}, w^{19}\right),\left(w^{11}, w\right),\left(w^{11}, w^{15}\right)\right\} .
\end{aligned}
$$

Note that all points removed from pentagons in $\mathcal{C}_{1}$ and $\mathcal{C}_{4}$ are in $\mathcal{F}_{19}$. A direct computation with Magma [3] shows that the corresponding 1-factorisation of the complete graph $K_{20}$ is rigid, that is, its only automorphism is the trivial one.

\subsection{Case $q=9$ (Theorem 2)}

Let $w$ be a primitive element of $\mathrm{GF}(81)$ such that $w^{4}=w^{3}+1$. Note that every nonzero element of $\mathrm{GF}(9)$ is a power of $w^{10}$, that is, $w^{10}$ is a primitive element of $\mathrm{GF}(9)$.

Consider the conic $\mathcal{C}$ of equation $Y=X^{2}$ in $\mathrm{PG}(2,81)$. There are 40 L-orbits: 9 short orbits, 27 good (long) orbits, and 4 bad (long) orbits. The points in bad orbits are 
contained in four conics $\mathcal{C}_{r}: Y=X^{2}-r$, where $r=1,2, w^{20}, w^{60}$. We need to adjust the construction from Section 3 because 9 is not a prime, the bad orbits split into 96 triangles (24 triangles from each bad orbits) and we do not have enough points in a unique short orbit. We provide an alternative construction that overcomes this difficulty.

The points on the 9 short orbits can be rearranged in the following 36-sets - again with the tangent property:

$$
L_{i}=E^{\prime} \cap \ell_{i}, i=0,1, \ldots, 8
$$

where $\ell_{0}$ is the vertical line of equation $X=0$ and $\ell_{i}$ is the vertical line of equation $X=w^{10 i}$ for $i=1, \ldots, 8$.

The sets $L_{1}, \ldots, L_{8}$ give rise to the first eight one-factors $F_{1}, \ldots, F_{8}$. The points in $L_{0}$ will be used, together with all the points from bad orbits, to form nine one-factors $F_{9}, \ldots, F_{17}$.

The set $L_{0}$ can be partitioned into nine $\Psi$-orbits:

$$
\begin{array}{lll}
S_{1}=\left(0, w^{2}\right)^{\Psi}, & S_{2}=\left(0, w^{4}\right)^{\Psi}, & S_{3}=\left(0, w^{6}\right)^{\Psi}, \\
S_{4}=\left(0, w^{8}\right)^{\Psi}, & S_{5}=\left(0, w^{10}\right)^{\Psi}, & S_{6}=\left(0, w^{12}\right)^{\Psi}, \\
S_{7}=\left(0, w^{14}\right)^{\Psi}, & S_{8}=\left(0, w^{16}\right)^{\Psi}, & S_{9}=\left(0, w^{18}\right)^{\Psi} .
\end{array}
$$

Each of these $\Psi$-orbit is a 4 -set with the tangent property.

Similarly, we can partition the points on bad orbits into $\Psi$-orbits

$$
\begin{array}{lll}
O_{1}=\left(w, w^{25}\right)^{\Psi}, & O_{2}=\left(w^{2}, w^{3}\right)^{\Psi}, & O_{3}=\left(w^{3}, w^{75}\right)^{\Psi}, \\
O_{4}=\left(w^{4}, w^{74}\right)^{\Psi}, & O_{5}=\left(w^{5}, w^{70}\right)^{\Psi}, & O_{6}=\left(w^{6}, w^{9}\right)^{\Psi}, \\
O_{7}=\left(w^{7}, w^{6}\right)^{\Psi}, & O_{8}=\left(w^{8}, w^{23}\right)^{\Psi}, & O_{9}=\left(w^{9}, w^{65}\right)^{\Psi}, \\
O_{10}=\left(w^{11}, w^{64}\right)^{\Psi}, & O_{11}=\left(w^{12}, w^{62}\right)^{\Psi}, & O_{12}=\left(w^{13}, w^{21}\right)^{\Psi}, \\
O_{13}=\left(w^{14}, w\right)^{\Psi}, & O_{14}=\left(w^{15}, w^{50}\right)^{\Psi}, & O_{15}=\left(w^{16}, w^{61}\right)^{\Psi}, \\
O_{16}=\left(w^{17}, w^{48}\right)^{\Psi}, & O_{17}=\left(w^{18}, w^{27}\right)^{\Psi}, & O_{18}=\left(w^{19}, w^{16}\right)^{\Psi}, \\
O_{19}=\left(w^{21}, w^{18}\right)^{\Psi}, & O_{20}=\left(w^{22}, w^{31}\right)^{\Psi}, & O_{21}=\left(w^{23}, w^{54}\right)^{\Psi}, \\
O_{22}=\left(w^{24}, w^{69}\right)^{\Psi}, & O_{23}=\left(w^{25}, w^{60}\right)^{\Psi}, & O_{24}=\left(w^{26}, w^{13}\right)^{\Psi}, \\
O_{25}=\left(w^{27}, w^{35}\right)^{\Psi}, & O_{26}=\left(w^{28}, w^{78}\right)^{\Psi}, & O_{27}=\left(w^{29}, w^{2}\right)^{\Psi}, \\
O_{28}=\left(w^{31}, w^{7}\right)^{\Psi}, & O_{29}=\left(w^{32}, w^{47}\right)^{\Psi}, & O_{30}=\left(w^{33}, w^{32}\right)^{\Psi}, \\
O_{31}=\left(w^{34}, w^{37}\right)^{\Psi}, & O_{32}=\left(w^{35}, w^{20}\right)^{\Psi}, & O_{33}=\left(w^{36}, w^{26}\right)^{\Psi}, \\
O_{34}=\left(w^{37}, w^{29}\right)^{\Psi}, & O_{35}=\left(w^{38}, w^{39}\right)^{\Psi}, & O_{36}=\left(w^{39}, w^{63}\right)^{\Psi} .
\end{array}
$$

Note that each of these $\Psi$-orbit is a 8 -set with the tangent property.

We can construct nine 36 -sets with the tangent property, each constructed as the union 
of four orbits $O_{i}$ and one orbit $S_{j}$. For instance, we can take:

$$
\begin{aligned}
& F_{9}=S_{1} \cup O_{1} \cup O_{6} \cup O_{9} \cup O_{25}, \\
& F_{10}=S_{2} \cup O_{2} \cup O_{3} \cup O_{11} \cup O_{36}, \\
& F_{11}=S_{3} \cup O_{7} \cup O_{18} \cup O_{21} \cup O_{33}, \\
& F_{12}=S_{4} \cup O_{17} \cup O_{26} \cup O_{31} \cup O_{35}, \\
& F_{13}=S_{5} \cup O_{12} \cup O_{14} \cup O_{27} \cup O_{29}, \\
& F_{14}=S_{6} \cup O_{8} \cup O_{15} \cup O_{24} \cup O_{30}, \\
& F_{15}=S_{7} \cup O_{4} \cup O_{10} \cup O_{23} \cup O_{32}, \\
& F_{16}=S_{8} \cup O_{5} \cup O_{16} \cup O_{19} \cup O_{20}, \\
& F_{17}=S_{9} \cup O_{13} \cup O_{22} \cup O_{28} \cup O_{34} .
\end{aligned}
$$

Note that the sets $F_{1}, \ldots, F_{17}$ are left invariant by the group $\Psi$ and cover all points in bad and short orbits. The last 54 one-factors can be obtained from good orbits using the construction from Section 3.

The full automorphism group of the one-factorisation so obtained can be easily computed with the aid of Magma [3]. It should be noticed that many of the good orbits split into two one-factors in several different ways, and thus produce different automorphism groups. Nevertheless, supported by some experiments, we expect the resulting onefactorisations to be rigid. For a matter of space, we are not providing all the details about this.

\section{Acknowledgements}

This research was carried out within the activities of the GNSAGA - Gruppo Nazionale per le Strutture Algebriche, Geometriche e le loro Applicazioni of the Italian INdAM.

Nicola Pace was supported by the Alexander von Humboldt Foundation with funds from the German Federal Ministry of Education and Research (BMBF).

\section{References}

[1] A. Bonisoli and G. Korchmáros. Suzuki groups, one-factorizations and Lüneburg planes. Discrete Math., 161(1-3):13-24, 1996.

[2] A. Bonisoli and D. Labbate. One-factorizations of complete graphs with vertexregular automorphism groups. J. Combin. Des., 10(1):1-16, 2002.

[3] W. Bosma, J.J. Cannon, and C. Playoust. The Magma algebra system. I. The user language. J. Symbolic Comput., 24(3-4):235-265, 1997.

[4] M. Buratti. Abelian 1-factorization of the complete graph. European J. Combin., 22(3):291-295, 2001.

[5] P. J. Cameron and G. Korchmáros. One-factorizations of complete graphs with a doubly transitive automorphism group. Bull. London Math. Soc., 25(1):1-6, 1993. 
[6] J. H. Dinitz and D. R. Stinson. Some new perfect one-factorizations from starters in finite fields. J. Graph Theory, 13(4):405-415, 1989.

[7] A. Hartman and A. Rosa. Cyclic one-factorization of the complete graph. European J. Combin., 6(1):45-48, 1985.

[8] J. W. P. Hirschfeld. Projective Geometries over Finite Fields. Oxford Mathematical Monographs. The Clarendon Press, Oxford University Press, New York, second edition, 1998.

[9] Gy. Kiss. One-factorizations of complete multigraphs and quadrics in $\mathrm{PG}(n, q)$. $J$. Combin. Des., 10(2):139-143, 2002.

[10] Gy. Kiss, N. Pace, and A. Sonnino. On circular-linear one-factorizations of the complete graph. Discrete Math., 342(12):Art. 11162, 2019.

[11] Gy. Kiss and C. Rubio-Montiel. A note on $m$-factorizations of complete multigraphs arising from designs. Ars Math. Contemp., 8(1):163-175, 2015.

[12] G. Korchmáros. Una proprietà gruppale delle involuzioni planari che mutano in sé un'ovale di un piano proiettivo finito. Ann. Mat. Pura Appl. (4), 116:189-205, 1978.

[13] G. Korchmáros. Cyclic one-factorization with an invariant one-factor of the complete graph. Ars Combin., 27:133-138, 1989.

[14] G. Korchmáros, N. Pace, and A. Sonnino. One-factorisations of complete graphs arising from ovals in finite planes. J. Combin. Theory Ser. A, 160:62-83, 2018.

[15] G. Korchmáros, A. Siciliano, and A. Sonnino. 1-factorizations of complete multigraphs arising from finite geometry. J. Combin. Theory Ser. A, 93(2):385-390, 2001.

[16] C. C. Lindner, E. Mendelsohn, and A. Rosa. On the number of 1-factorizations of the complete graph. J. Combinatorial Theory Ser. B, 20(3):265-282, 1976.

[17] E. Mendelsohn and A. Rosa. One-factorizations of the complete graph-a survey. J. Graph Theory, 9(1):43-65, 1985.

[18] N. Pace and A. Sonnino. One-factorisations of complete graphs arising from hyperbolae in the Desarguesian affine plane. J. Geom., 110(1):Art. 15, 19 pp., 2019.

[19] A. Sonnino. One-factorizations of complete multigraphs arising from maximal $(k ; n)$ arcs in PG(2, $\left.2^{h}\right)$. Discrete Math., 231(1-3):447-451, 2001.

[20] W. D. Wallis. On one-factorizations of complete graphs. J. Austral. Math. Soc., 16:167-171, 1973. Collection of articles dedicated to the memory of Hanna Neumann, II.

[21] W. D. Wallis. One-factorizations of complete graphs. In Contemporary design theory, Wiley-Intersci. Ser. Discrete Math. Optim., pages 593-631. Wiley, New York, 1992.

[22] W. D. Wallis. One-factorizations, volume 390 of Mathematics and its Applications. Kluwer Academic Publishers Group, Dordrecht, 1997. 